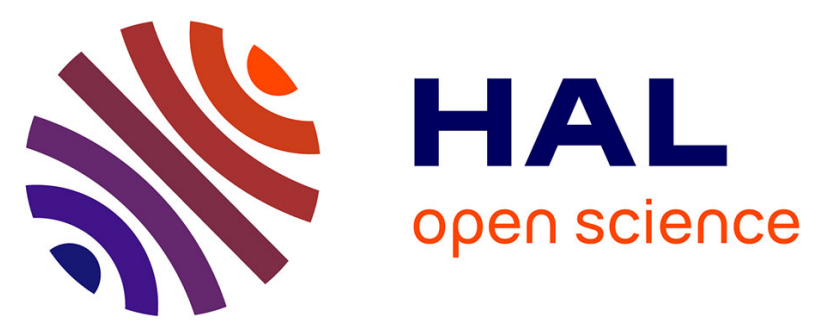

\title{
Formal Concept Analysis for the Interpretation of Relational Learning applied on 3D Protein-Binding Sites
}

Emmanuel Bresso, Renaud Grisoni, Marie-Dominique Devignes, Amedeo

Napoli, Malika Smail-Tabbone

\section{- To cite this version:}

Emmanuel Bresso, Renaud Grisoni, Marie-Dominique Devignes, Amedeo Napoli, Malika SmailTabbone. Formal Concept Analysis for the Interpretation of Relational Learning applied on 3D Protein-Binding Sites. 4th international conference on Knowledge Discovery and Information Retrieval - KDIR 2012, Oct 2012, Barcelona, Spain. 12 p. hal-00734349

\author{
HAL Id: hal-00734349 \\ https://hal.inria.fr/hal-00734349
}

Submitted on 21 Oct 2012

HAL is a multi-disciplinary open access archive for the deposit and dissemination of scientific research documents, whether they are published or not. The documents may come from teaching and research institutions in France or abroad, or from public or private research centers.
L'archive ouverte pluridisciplinaire HAL, est destinée au dépôt et à la diffusion de documents scientifiques de niveau recherche, publiés ou non, émanant des établissements d'enseignement et de recherche français ou étrangers, des laboratoires publics ou privés. 


\title{
Formal Concept Analysis for the Interpretation of Relational Learning applied on 3D Protein-Binding Sites
}

\author{
Emmanuel BreSSO ${ }^{1,2}$, Renaud GRISONI ${ }^{2}$, Marie-Dominique DEVIGNES ${ }^{1,2,3}$, Amedeo NAPOLI ${ }^{1,2,3}$ and \\ Malika SMAIL-TABBONE ${ }^{1,2}$ \\ ${ }^{1}$ Université de Lorraine, LORIA, UMR 7503, Vandoeuvre-les-Nancy, F-54506, France \\ ${ }^{2}$ Inria, Villers-lès-Nancy, F-54600, France \\ ${ }^{3}$ CNRS, LORIA, UMR 7503, Vandoeuvre-les-Nancy, F-54506, France \\ \{bressoem, rgrisoni, devignes, napoli, malika\}@loria.fr
}

Keywords: Inductive Logic Programme: Formal Concept Analysis: Knowledge Discovery: 3D Protein Binding Sites

Abstract: Inductive Logic Programming (ILP) is a powerful learning method which allows an expressive representation of the data and produces explicit knowledge. However, ILP systems suffer from a major drawback as they return a single theory based on heuristic user-choices of various parameters, thus ignoring potentially relevant rules. Accordingly, we propose an original approach based on Formal Concept Analysis for effective interpretation of reached theories with the possibility of adding domain knowledge. Our approach is applied to the characterization of three-dimensional (3D) protein-binding sites which are the protein portions on which interactions with other proteins take place. In this context, we define a relational and logical representation of $3 \mathrm{D}$ patches and formalize the problem as a concept learning problem using ILP. We report here the results we obtained on a particular category of protein-binding sites namely phosphorylation sites using ILP followed by FCA-based interpretation.

\section{INTRODUCTION AND MOTIVATION}

Relational or logical learning is currently one of the most promising research topics in knowledge discovery, especially for complex application domains (De Raedt, 2008). Life sciences provide a wide variety of such applications. In our work we investigate how relational learning can contribute to the understanding of proteinprotein interactions which are important for most cellular processes. Great effort has been put into both experimental and computational methods to identify or predict protein-protein interactions. In protein docking, geometric and steric considerations are used to fit two protein structures into a bound complex (Smith et al., 2002). Alternative computational methods predict bindings between pairs of proteins based either on their homology with known binding pairs of proteins or on integrated data from a wide variety of sources (Aloy and Russel, 2003; Jansen et al., 2003; Tran et al., 2005). However, despite the large number of reported computational methods, precise understanding of protein-protein interactions still raises various challenges.

On the one hand, most reported methods for structure-based prediction of protein-protein interactions apply on a single data table where each interaction site (or interface) is described by a set of descriptors or attributes including diverse physico-chemical properties aggregated on the whole binding site such as the residue composition, hydrophobicity, accessible surface area (Jones and Thornton, 1997; Zhu et al., 2006). However, this data model prevents from representing individual properties of the interface components (accessible surface of a particular residue) or spatial relations between components (e.g., distance between two residues). Hence, more expressive languages are necessary to represent the structural aspect of 3D interaction sites. Such sites are called hereafter Protein-Binding Sites (PBS) to make a clear distinction from ligand-binding sites on protein surface.

On the other hand, most current methods do not provide explicit characterization of PBS along with the prediction model. For instance, methods based on Support Vector Machines (SVM) act as black-boxes returning 
outputs with respect to inputs without explanation (Zhu et al., 2006). Explicit characterization of PBS would obviously provide good insights of the underlying biological phenomena. In this context our aim is to exploit the growing set of available protein 3D structures for characterizing PBS and go beyond the limitations of the most current approaches qualified as black-box and single-table. To achieve this objective, we propose to apply a ILP (Inductive Logic Programming) learning method on the descriptions of protein 3D patches in order to induce a general definition of the PBS concept. Our approach includes a logical (relational) representation of protein 3D patches corresponding to positive or negative examples of PBS.

Inductive Logic Programming (ILP) allows to learn a concept definition from observations, i.e., a set of positive examples (E+) and a set of negative examples (E-), and background knowledge (B) (Muggleton, 1991). Given E+, E-, and B the goal of ILP is to induce a set of rules or a theory T that is consistent (T UB covers or explains each positive example in $\mathrm{E}+$ ), and complete ( $\mathrm{T} \cup \mathrm{B}$ does not cover or contradicts any negative example in E-).

In most ILP systems both B and T are represented as definite clauses (or prolog programs) in First-Order Logic (FOL), i.e., a disjunction of literals with one positive literal. A rule has the form "head :- body" and is interpreted as: if the conditions in the body are true then the head is true as a logical consequence. The background knowledge B includes (i) the relational description of the examples using a set of relevant n-ary predicates and (ii) a priori domain knowledge, i.e., a set of rules and facts which don't refer to any example but express what is known about the elements which describe the examples. The theory $\mathrm{T}$ is a set of rules which cover as many of the positive examples as possible and the fewest negative examples. The head of each rule is the concept to learn whereas the body contains the induced description of the concept (generalization of examples). The rule search is performed in a clause space where the clause subsumption allows building generalizations or specializations of the clauses (Muggleton and De Raedt, 1994). As the clause space is too large to be exhaustively explored, heuristic mechanisms exist to reduce its size and make the induction process feasible. These mechanisms allow the user to define which kind of rules (s)he wants to get. These learning biases are defined by setting some parameters that influence the search strategy and lead to different theories. Consequently, albeit ILP systems have important properties that make them good candidates for knowledge discovery tasks in complex domains, they have a major shortcoming which may impede their usability: a single theory based on heuristics is returned, thus ignoring many clauses that may be relevant for the domain expert (Page and Srinivasan 2003). This may partly explain the low impact of the logical learning noticed by King (2011). Consequently, we propose in this paper an original approach to improve the ILP-based knowledge discovery thanks to classification-based interpretation of different theories. A binary table is built according to the covering relation between examples and rules. Formal Concept Analysis (FCA, Ganter and Wille, 1999) is used to identify in the table formal concepts grouping examples covered by the same rules.

For a first validation of our approach, we chose a specific group of PBS, the phosphorylation sites. Indeed, phosphorylation is an important biological process and phosphorylation sites are exhaustively listed in a unique data source (Diella et al., 2008). The rest of the paper is organized as follows. Section 2 introduces the knowledge discovery problem and the ILP program we used along with the different experimental settings. Section 3 describes our proposal for theory interpretation using FCA and domain knowledge. Section 4 summarizes the results obtained with our application on PBS. We discuss our results and describe related work in Section 5.

\section{ILP FOR CHARACTERISING 3D PROTEIN-BINDING SITES}

\subsection{The data representation language}

Protein 3D Patch Definition: Protein surface patches were first introduced by Jones and Thornton (1997) who defined a surface patch as a central surface accessible residue (amino acid) along with the nearest surface accessible neighbours. For our part, we define a protein $3 \mathrm{D}$ patch as a spherical fragment of a protein $3 \mathrm{D}$ structure centred on a residue of the protein, the central core residue (Guharoy and Chakrabarti, 2005). A 3D patch has a radius $r$ corresponding to the sphere radius and the residues composing the patch are those having an atom whose distance to the central residue does not exceed r. The RCSB PDB database (www.pdb.org) stores the resolved 3D structures of proteins (Berman et al., 2000). 
Protein 3D Patch descriptors: We propose to describe a 3D patch at two levels: (i) the patch is characterized as a whole by a set of global descriptors such as patch solvent accessible surface area (ASA), the number of carbon atoms occurring in the patch, and the number of residues in the patch; (ii) the patch composition and structure are characterized by a set of descriptors describing secondary and tertiary structure information on the patch residues. At the latter level, each residue of the patch is described by its name and its relative position in the primary sequence of the protein with respect to the central residue of the patch. The ASA value of each residue is used as a local descriptor of the residue in the patch. Two descriptors indicate if a given residue is on a helix, respectively on a sheet. Finally, one descriptor represents the spatial distance between each patch residue and the central residue. This distance information may play an important role in the interaction building. Figure 1 shows an example of a protein 3D patch. The variety of relationships between the elements of a patch clearly requires a relational or logical representation language as a feature-based language could only represent the global descriptors of the patches.

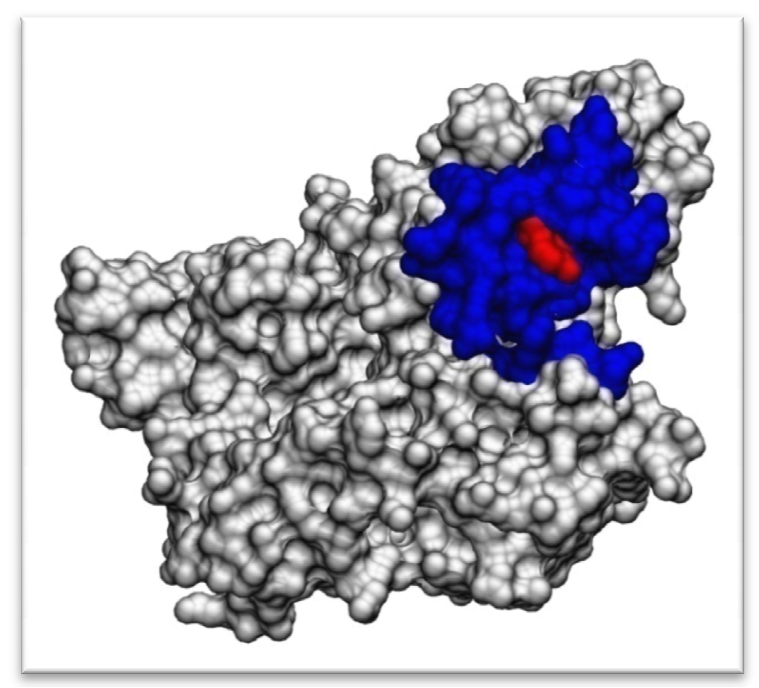

Figure 1: Visualization of a protein (PDB ID: 1opk) 3D patch using the VMD program (Humphrey et al., 1996). The central residue of the patch is represented in red. The patch surface accessible to the solvent is represented in blue.

Characterization of $3 D$ PBS as a relational learning problem: Our objective is to learn with ILP a definition of the Protein-Binding Site concept given relational descriptions of positive and negative examples of this concept.

We define a set of first-order predicates relevant for the ILP problem (Table 1). The unary predicate "pbs" is the concept to learn and has a 3D patch identifier as argument. Several binary predicates represent the global descriptors of the patch such as the predicates "p_asa" and "p_c" which correspond respectively to the ASA value of a patch and the number of carbon atoms. A set of ternary and 4-ary predicates represent the structural descriptors of the 3D patches. One 4-ary predicate is "p_r_distance" which represents the distance value of a patch residue from the central residue. One ternary predicate is "p_r_helix" which expresses that a patch residue belongs to a helix. A supplementary ternary predicate is "p_r_surface" which is derived from the "p_r_asa" predicate. Finally, a prolog rule allows to infer that a residue is on the patch surface if its ASA value is greater than the threshold $10 \AA^{2}$ :

p_r_surface (pid,res,pos) :- p_r_asa (pid,res,pos,v), greater_than $(v, 10)$.

As mentioned before, ILP allows to use domain knowledge during the induction process. In our case, there is a consensus to classify the different residues in several classes reflecting shared physico-chemical properties which may play a role in protein-protein interfaces (Jones and Thornton, 1997). Hence, we choose to use two classifications as a priori domain knowledge (Table 2). Consequently, we define a unary predicate for each residue class (e.g., acidic, basic) whose interpretation is defined according to the class membership (e.g., acidic(asp), basic(arg)).

Table 1: First order-logic predicates to describe protein 3D patches 


\begin{tabular}{|l|l|}
\hline Predicate & Interpretation \\
\hline pbs (pid) & $\begin{array}{l}\text { The patch identified by pid is a } \\
\text { protein-binding site }\end{array}$ \\
\hline p_asa (pid, $v)$ & $\begin{array}{l}\text { v is the solvent accessible } \\
\text { surface value of the pid patch }\end{array}$ \\
\hline $\begin{array}{l}\text { p_c (pid,n), p_o (pid, } n), \\
\text { p_n (pid,n), p_s (pid, } n \text { ) }\end{array}$ & $\begin{array}{l}\mathrm{n} \text { is the number of } \\
\text { carbon/oxygen/nitrogen/sulphur } \\
\text { atoms in the pid patch }\end{array}$ \\
\hline p_r (pid,res,pos) & $\begin{array}{l}\text { res is the name of the residue at } \\
\text { relative position pos in the pid } \\
\text { patch }\end{array}$ \\
\hline p_r_asa (pid,res,pos,v) & $\begin{array}{l}\text { vis the ASA value of the } \\
\text { residue named res at the relative } \\
\text { position pos (in the primary } \\
\text { sequence) in the pid patch }\end{array}$ \\
\hline $\begin{array}{l}\text { p_r_distance } \\
\text { (pid,res,pos,v) }\end{array}$ & $\begin{array}{l}\text { v is the spatial distance between } \\
\text { the residue (res, pos) and the } \\
\text { central residue in the pid patch }\end{array}$ \\
\hline $\begin{array}{l}\text { p_r_helix (pid,res,pos), } \\
\text { p_r_sheet (pid,res,pos) }\end{array}$ & $\begin{array}{l}\text { the residue (res, pos) is on a } \\
\text { helix/sheet in the pid patch }\end{array}$ \\
\hline
\end{tabular}

Table 2: Physico-chemical classes of residues defined in (Yu et al., 2006) and (Dubchak et al., 1999).

\begin{tabular}{|l|l|}
\hline \multicolumn{1}{|c|}{ Class Name } & \multicolumn{1}{c|}{ Residues in the Class } \\
\hline acidic & asp, glu \\
\hline basic & arg., hais, lys \\
\hline aromatic & phe, trp, yr \\
\hline amide & asn, gln \\
\hline small hydroxyl & ser, thr \\
\hline sulphur containing & cys, met \\
\hline aliphatic1 & ala, gly, pro \\
\hline aliphatic2 & ile, leu, val \\
\hline aliphatic & ala, gly, pro, ile, leu, val \\
\hline small & gly,ala,ser,cys, thr,pro,asp \\
\hline medium & asn, val, glu, gln, ile, leu \\
\hline large & met, his, lys, phe, arg, tyr, trp \\
\hline low polarizability & gly, ala, ser, asp, thr \\
\hline medium polarizability & cys, pro, asn, val, glu, gln, ile, leu \\
\hline high polarizability & lys, met, his, phe, arg, tyr, trp \\
\hline hydrophobic & cys, val, leu, ile, met, phe, trp \\
\hline
\end{tabular}

To sum up, the global and structural descriptors are computed for a set of 3D patches and represented with respect to the defined FOL predicates, forming a learning set. A ILP program can then be used to learn FOL rules characterizing or covering subsets of positive patches.

\subsection{The ILP program and its parameters}

The experiments reported in this paper were conducted with the Aleph program whose basic algorithm is described in four steps (Srinivasan, 2007):

1. Select a seed example to be generalized. If none exists, stop. 
2. Construct the most specific clause that entails the example selected, and is compliant with the language restrictions provided. This is usually a definite clause with many literals, and is called the "bottom clause".

3. Find a clause more general than the bottom clause. This is done by searching for some subset of the literals in the bottom clause that has the "best" evaluation score.

4. The clause with the best score is added as a rule to the current theory, and all examples made redundant are removed. Return to Step 1.

Many parameters can be set for tuning some aspect of the theory construction with Aleph. For instance, the rule evaluation function can be chosen and the default one is based on the difference between the number of covered positive examples and the number of covered negative examples. The noise parameter is the maximum negative examples that an acceptable rule may cover (default value is 0 ). This parameter can be set to higher values in case of noisy data (in our study, one is never sure that a negative PBS is a true one unless experimental evidence is provided). The min-pos parameter is the minimal number of positive examples that a rule must cover (default value is 1). Aleph also requires other learning biases to be defined as (i) a set of determinations defines the predicate to learn and the predicates which can appear in the rules; (ii) a set of modes defines the types of predicate arguments and the way they can be chained in a rule.

As the above algorithm suggests, Aleph iterates on the positive examples of the learning set for building the most specific clause of a chosen seed example which is compliant with the defined bias and covers the maximum number of positive examples and the minimum number of negative ones. When finding the best rule, the examples covered by the best rule may be removed or not from the seed set and/or from the learning set (used for the rule evaluation). Hence with regard to this removal step, a induce-type parameter defines three ways of theory construction, (i) induce (covered examples are removed from both the seed set and the learning set), (ii) induce-cover (covered examples are removed from the seed set and not from the learning set), and (iii) induce-max (covered examples are removed neither from the seed set nor the learning set). Consequently, both induce and induce-cover are sensitive to the order in which the seed examples are presented contrasting with induce-max (each example is generalized). Both induce-cover and induce-max produce rules with more overlap than induce.

\section{FCA-BASED INTERPRETATION OF A THEORY}

Different theories are reached by the ILP program depending on the values of the program parameters set by the user in a heuristic way. It is then important to allow the domain expert to explore each theory. Each theory is globally characterized by the learning set coverage, i.e., the proportion of positive examples that are covered by at least one rule of the theory (theory coverage). Another global criterion is the coverage of the best rule. Furthermore, we propose a FCA-based analysis of the ILP learning results including or not domain knowledge to help the expert in the interpretation task. This can possibly lead to one or more preferred theories.

\subsection{Formal Concept Analysis (FCA)}

The framework of FCA is fully detailed in (Ganter and Wille, 1999). FCA starts with a formal context (G,M,I) where $\mathrm{G}$ denotes a set of objects, $\mathrm{M}$ a set of attributes, and $\mathrm{I} \subseteq \mathrm{G} \times \mathrm{M}$ a binary relation between $\mathrm{G}$ and $\mathrm{M}$. The statement $(g, m) \in \mathrm{I}$ is interpreted as "the object $g$ has attribute $m$ " (also noted $g I m$ ). Two operators (.)' define a Galois connection between the powersets $\left(2^{\mathrm{G}}, \subseteq\right)$ and $\left(2^{\mathrm{M}}, \subseteq\right)$, with $\mathrm{A} \subseteq \mathrm{G}$ and $\mathrm{B} \subseteq \mathrm{M}$ :

$A^{\prime}=\{m \in M \mid \forall g \in A: g \operatorname{Im}\}$ and

$B^{\prime}=\{g \in G \mid \forall m \in B: g I m\}$

For $\mathrm{A} \subseteq \mathrm{G}, \mathrm{B} \subseteq \mathrm{M}$, a pair $(\mathrm{A}, \mathrm{B})$, such that $\mathrm{A}^{\prime}=\mathrm{B}$ and $\mathrm{B}^{\prime}=\mathrm{A}$, is called a formal concept. In $(\mathrm{A}, \mathrm{B})$, the set $\mathrm{A}$ is called the extent and the set $\mathrm{B}$ the intent of the concept $(\mathrm{A}, \mathrm{B})$. Formal concepts are partially ordered by the concept subsumption:

$(\mathrm{A} 1, \mathrm{~B} 1) \leq(\mathrm{A} 2, \mathrm{~B} 2) \Leftrightarrow \mathrm{A} 1 \subseteq \mathrm{A} 2 \Leftrightarrow \mathrm{B} 2 \subseteq \mathrm{B} 1$. With respect to this partial order, the set of all formal concepts forms a full lattice called the concept lattice of (G,M,I). The FCA implementation used in our experiments was the one of the Coron data mining platform (Szathmary, 2006)

\subsection{FCA-based analysis of a ILP theory}


A first interpretation of a theory is defined on the concept lattice issued from FCA applied on the binary patchrule matrix describing the coverage of positive examples by the rules (only the body part of the rules is necessary since the head is the same for all rules). Table 3 shows an example of a formal context with 8 patches and 3 rules. In our case, a formal concept gathers a subgroup of 3D patches which are covered by the same set of rules. Figure 2 shows the concept lattice obtained from the context presented in Table 3 . The concept lattice forms a good browsing means in the ILP results allowing the expert to move from a set of patches covered by one rule to more specific concepts containing subsets of the patches covered by two rules, three rules and so on. Indeed, it is relevant to count for each theory, the number of concepts of more than two rules (multiple-rule concepts) and having a significant extent size (number of patches). Furthermore, multiple-rule concepts can be examined by the domain expert to see if any concept intent (rule conjunction) provides a relevant description of the corresponding patch subgroup.

A second interpretation of a theory is defined on the results of FCA applied to the patch-rule covering matrix enriched with supplementary domain properties of the examples that weren't used in the learning process. This allows the expert to analyze how the theory rules are related to known properties of the examples. More precisely, interesting concepts to look at are those whose intent includes specific properties along with rules and whose extent has a significant size. These formal concepts gather 3D patches sharing domain property(ies) and being covered by the same rule(s). Involved rule(s) will be said to be associated with the domain property(ies).

\section{RESULTS}

As a first validation of our approach, we apply it to particular category of protein-binding sites, i.e., phosphorylation sites. We first present the learning set we established (Section 4.1) and the results of the FCAbased interpretation of 18 theories we have achieved using the Aleph program (Section 4.2).

\subsection{The learning dataset of the case study}

Phosphorylation is a reversible post-translation modification of a protein due to a kinase adding a phosphate group to a serine, threonine, or a tyrosine residue (so called phosphorylated residue). A set of experimentally verified eukaryotic Phosphorylation Sites (PS) is available in the Phospho.ELM database (Diella et al., 2008). In this study, we build a learning set including all known phosphorylation sites irrespective of the phosphorylating kinase and the phosphorylated residue. This contrasts with the systems which perform the prediction of residuespecific or kinase-specific phosphorylation sites (Wong et al., 2007; Durek et al., 2009). The PS concern about 5976 distinct proteins among which only 286 have exploitable 3D structures. The PS relative to those proteins divide into 231 serine sites, 90 threonine sites, and 193 tyrosine sites. For each PS we extract one positive 3D patch from the PDB best-resolution 3D structure of the corresponding protein. This patch is centered on the phosphorylated residue (serine, threonine, or tyrosine). As for negative examples, given a protein structure for which PS are known, biologists are able to identify with reasonable confidence 3D patches corresponding to negative PS. Thus, a negative 3D patch is built for each serine/threonine/tyrosine residue which is not known as a phosphorylable residue and whose minimal spatial distance to a phosphorylable residue is reasonably high (greater than $10 \AA$ in our experiments). The total number of found negative examples is 687 representing $57 \%$ of the learning dataset. The proposed descriptors for protein patches are computed on the basis of the $3 \mathrm{D}$ coordinates of the patch atoms extracted from the PDB structures. The numeric descriptor values were discretized by equal-frequency binning.

Table 3: Formal context example

\begin{tabular}{|l|c|c|c|}
\hline & rule_1 & rule_2 & rule_3 \\
\hline patch_1 & $\mathrm{x}$ & & $\mathrm{x}$ \\
\hline patch_2 & & & \\
\hline patch_3 & $\mathrm{x}$ & $\mathrm{x}$ & \\
\hline patch_4 & $\mathrm{x}$ & $\mathrm{x}$ & $\mathrm{x}$ \\
\hline patch_5 & & $\mathrm{x}$ & \\
\hline patch_6 & $\mathrm{x}$ & & $\mathrm{x}$ \\
\hline patch_7 & & $\mathrm{x}$ & $\mathrm{x}$ \\
\hline patch_8 & & & \\
\hline
\end{tabular}


Our objective is the 3D PBS characterization instead of their prediction especially as there exist for the phosphorylation case sequence-based prediction programs which exhibit good performances (Wong et al., 2007). Besides, the amount of available protein 3D structures is fairly smaller than the available protein sequences making it difficult to reach the prediction accuracy of those programs. Nevertheless, the prediction accuracy of a ILP theory (defined as the number of true positives plus true negatives over the total number of examples) is interesting to consider for building the learning set and comparing different theories. Hence theory prediction accuracy was computed by a tenfold cross-validation test for variable negative example percentages in the learning set (induce type and min-pos are set to induce-cover and 13). The results are given in Table 4 and confirm that the prediction accuracy continuously increases with the percentage of negative example. It is thus relevant to use the whole set of found negative 3D patches (i.e., 57\% negative examples versus $43 \%$ positive examples).

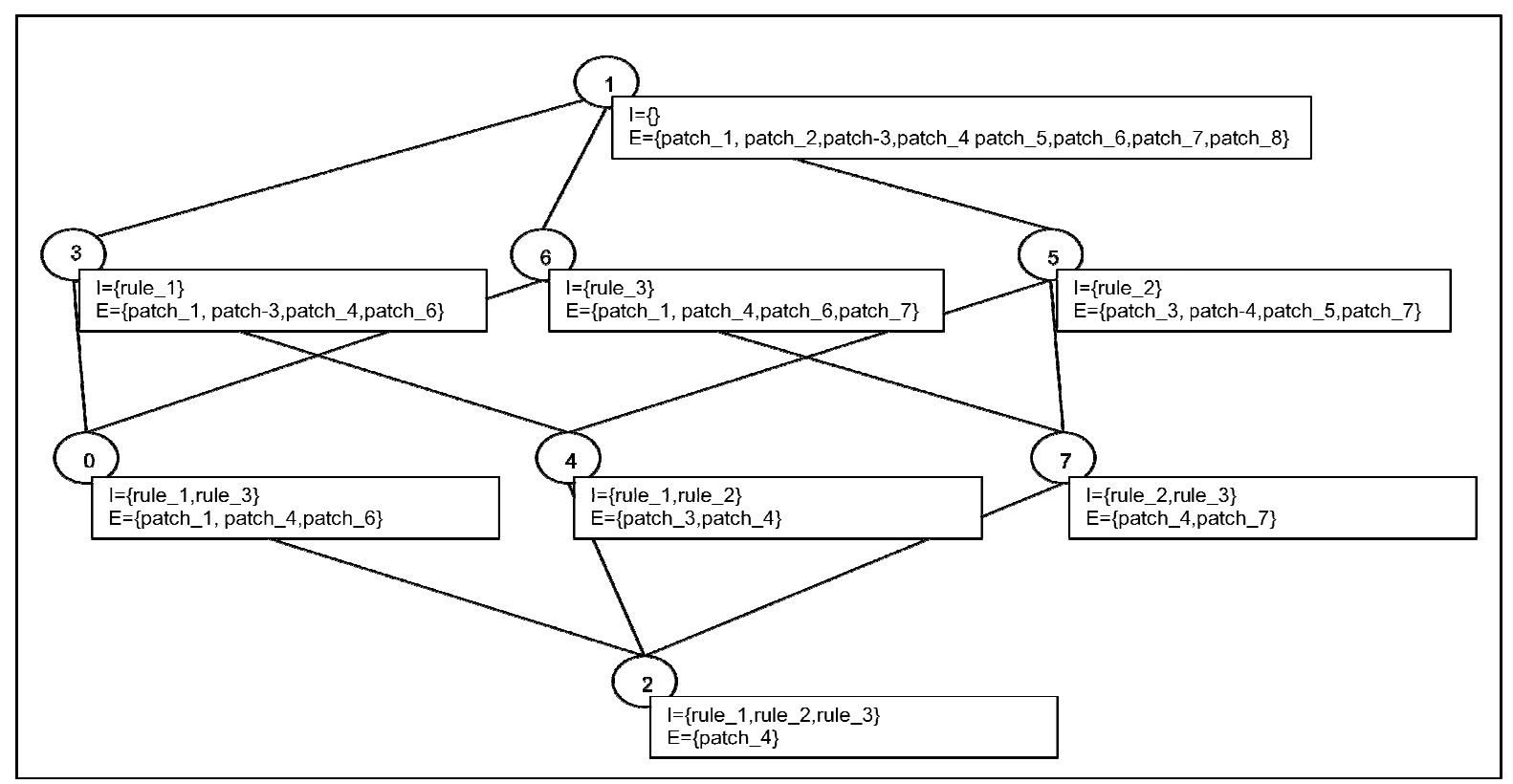

Figure 2: The concept lattice obtained by FCA from Table 3. Each numbered concept has an intent (I) and an extent (E). The edges correspond to subsumption relationships.

\subsection{FCA-based interpretation of ILP theories}

In our experiments, we consider three parameters which alter the way the rule search is seeded and the rules are selected in the Aleph program: induce-type (induce, induce-cover, induce-max), min-pos (from 8 to 12), and noise ( 0 or 1$)$. The rest of the parameters were set to the default value. In order to reduce the number of theories to interpret, we used as theory ranking criterion the prediction accuracy value (tenfold cross validated). We selected the 18 theories having a prediction value greater than $60 \%$. In fact the accuracy values vary from 54 to $63 \%$. As mentioned before the relatively weak values of prediction accuracy can be explained by the small size of the training set due to the small number of currently available 3D structures and its heterogeneity with respect to the phosphorylated residue and the phosphorylating kinase. The global criteria and the quantitative results of the FCA-based interpretation of the 18 selected theories are reported in Table 5. We notice that, the parameters have a combined effect on the theory size, the theory coverage and the best rule coverage as well as on the concept lattice composition. The most obvious trend is the continuous decrease of theory size, theory coverage and the number of multiple-rule concepts when increasing min-pos for a given induce-type and noise value. This makes the choice of an optimal configuration difficult because some trade-off must be found between these 
criteria. For instance, a small size is expected for a valuable theory but this should not be at the expense of its coverage.

Table 4: Prediction accuracy values (tenfold cross validated) obtained when varying the negative example percentage in the learning set.

\begin{tabular}{|l|l|l|}
\hline $\begin{array}{l}\text { Percentage of } \\
\text { negative examples }\end{array}$ & $\begin{array}{l}\text { Noise } \\
\text { parameter }\end{array}$ & $\begin{array}{l}\text { Prediction } \\
\text { accuracy }\end{array}$ \\
\hline 35 & 0 & 52 \\
\hline 35 & 1 & 54 \\
\hline 40 & 0 & 54 \\
\hline 40 & 1 & 55 \\
\hline 45 & 0 & 54 \\
\hline 45 & 1 & 55 \\
\hline 50 & 0 & 55 \\
\hline 50 & 1 & 57 \\
\hline 55 & 0 & 58 \\
\hline 55 & 1 & 58 \\
\hline 57 & 0 & 59 \\
\hline 57 & 1 & 61 \\
\hline
\end{tabular}

Table 5: Quantitative results of the FCA applied on the patch-rule covering matrices for the 18 selected theories.

\begin{tabular}{|l|l|l|l|}
\hline $\begin{array}{l}\text { Theory } \\
\text { parameters } \\
\text { (induce-type/ } \\
\text { min-pos) }\end{array}$ & $\begin{array}{l}\text { Theory } \\
\text { size / best } \\
\text { rule } \\
\text { coverage }\end{array}$ & $\begin{array}{l}\text { Theory } \\
\text { coverage } \\
(\%)\end{array}$ & $\begin{array}{l}\text { \# multiple- } \\
\text { rule } \\
\text { concepts/ } \\
\text { best concept } \\
\text { extent size }\end{array}$ \\
\hline noise=0 & \multicolumn{5}{|l|}{} \\
\hline induce-cover/9 & $64 / 15$ & 56 & $106 / 9$ \\
\hline induce-cover/10 & $37 / 16$ & 39 & $71 / 14$ \\
\hline induce-max /9 & $64 / 15$ & 56 & $106 / 9$ \\
\hline induce-max/10 & $39 / 16$ & 39 & $88 / 14$ \\
\hline noise=1 & $26 / 17$ & 48 & $8 / 3$ \\
\hline induce/8 & $19 / 17$ & 39 & $5 / 3$ \\
\hline induce/9 & $14 / 16$ & 32 & $3 / 3$ \\
\hline induce /10 & $10 / 15$ & 24 & $0 / 0$ \\
\hline induce /11 & $85 / 18$ & 73 & $215 / 13$ \\
\hline induce-cover/10 & $58 / 18$ & 61 & $144 / 16$ \\
\hline induce-cover/11 & $58 / 18$ & $91 / 13$ \\
\hline induce-cover/12 & $38 / 18$ & 48 & $50 / 13$ \\
\hline induce-cover/13 & $20 / 18$ & 30 & $31 / 16$ \\
\hline induce-cover/14 & $15 / 18$ & 23 & $386 / 16$ \\
\hline induce-max/10 & $101 / 18$ & 73 & $254 / 16$ \\
\hline induce-max/11 & $69 / 18$ & 61 & $136 / 16$ \\
\hline induce-max/12 & $43 / 18$ & 48 & $80 / 16$ \\
\hline induce-max/13 & $24 / 18$ & 30 & $31 / 16$ \\
\hline induce-max/14 & $15 / 18$ & 23 & \\
\hline
\end{tabular}

To illustrate the effect of the induce-type parameter on the clause search, the theory reached with the induce/10/1 configuration is provided (Appendix). One rule found in a different configuration (induce$\max / 10 / 1)$ and missing in the previous theory is the following:

$\operatorname{pbs}(A)$ :- $p_{-}$r_helix(A,B,3), high_polarizability(B), p_r $(A, \operatorname{pro}, 1)$.

This rule covers 17 positive examples but is not retrieved when switching the induce-type parameter from induce-max to induce.

To illustrate the browsing process in the concept lattice, we examined multiple-rule concepts from the 18 lattices. For instance, the theory with induce-max/12/1 produces a formal concept of 14 patches sharing the two following rules: 
$\operatorname{pbs}(A)$ :- p_r_surface $(A, B, 0)$, p_r_helix $(A, C, 21)$, p_r_helix $(A, D, 28)$.

$\operatorname{pbs}(A)$ :- p_r_surface $(A, B, 0), p_{-}$__helix $(A, C, 17)$, p_r_helix(A,D,28).

When browsing up in the lattice, we find a subgroup composed of 11 patches which share the two previous rules besides the following third one:

pbs(A) :- p_r_surface $(A, B, 0)$, p_r_helix $(A, C, 20)$, p_r_helix $(A, D, 28)$.

Hence, this subgroup of 11 patches is better characterized by the conjunction of the three rule bodies (after variable renaming and removal of redundant literals):

p_r_surface $(A, B, 0), p_{-}$__helix $(A, C, 21), p_{-}$_r_helix $(A, D, 28), p_{-} r$ helix $(A, E, 17), p_{-}$_h helix $(A, F, 20)$.

In more general terms, selecting formal concepts with multiple rules constitutes an interesting way to achieve longer rules than in the theory issued by Aleph. Indeed, the compression heuristic used during the clause search leads most ILP programs to favor shorter clauses to longer ones. Finn et al., (1998) proposed a solution to this drawback suitable to the pharmacophore search case.

An additional way to explore the concept lattices is to count how many multiple-rule concepts are enriched in specific patches with respect to the phosphorylated residue (serine or threonine versus tyrosine). Over the 18 analysed lattices we observed that more than $75 \%$ of the multiple-rule concepts are either tyrosine specific or serine-or-threonine specific. This provides the expert with relevant descriptions for each type of phosphorylation sites as well as descriptions common to both types.

\subsection{FCA-based interpretation of ILP theories including domain knowledge}

We applied FCA on the patch-rule table enriched with supplementary domain properties of the examples not used in the learning process, namely the phosphorylating kinase and the functional domain on which the 3D patch is located (Punta et al., 2012). ILP produces a set of rules covering 3D patches and the interpretation procedure we propose here helps the expert to analyze how the rules are related to known properties of the examples. About 50 kinases phosphorylate more than 2 patches of the learning set whereas 40 Pfam domains are associated to more than 2 patches. The patch-rule table is thus updated with those kinases and functional domains as supplementary properties of the patches. Some formal concepts of the resulting lattice include at least one rule along with a kinase (respectively a Pfam domain) and whose extent contains more than $1 / 3$ of the patches associated to the kinase (respectively the Pfam domain). The rules involved in such concepts can be examined by the expert in order to check their consistency with previous knowledge and whether they reveal novel relevant knowledge units regarding the kinase (respectively Pfam domain) concerned. For example the following rule was found associated with PKB kinases in concepts grouping the majority of 3D patches phosphorylated by this type of kinases:

pbs(A) :- p_r_helix(A,B,-4), p_r_helix(A,arg,-3), p_r_helix(A,ser,0).

In this rule the expert recognizes in particular the fact that an arginine residue is present at position -3 which is a well established observation for PKB kinases (Obata et al., 2000). More precisely the second literal expresses that the arginine residue at -3 position should be on a helix, which reveals to be interesting for characterizing those 3D patches.

Quantitative analysis of domain-knowledge FCA was performed in order to help the expert assessing the relative relevance of a theory with respect to another one. To this aim, the number of kinases (respectively Pfam domains) appearing at least once in a concept with an extent containing at least $1 / 3$ of the concerned patches was computed for each of the 18 theories selected in this study. The results are presented in Table 6 . Interestingly, it appears that, in particular with induce-max, the number of kinases does not continuously decrease with increasing values of the min-pos parameter. This contrasts with the continuous decrease of the theory coverage and size (Table 5). Thus even at low coverage values, the theory rules still exhibit descriptive ability correlated with domain knowledge. One can notice that such low-coverage theories would be discarded if the objective was prediction instead of characterization. 
Table 6: Number of kinases (respectively Pfam domains) appearing in concepts whose extent contains more than 1/3 of the patches associated to the kinase (respectively Pfam domain).

\begin{tabular}{|l|l|l|}
\hline $\begin{array}{l}\text { Theory parameters } \\
\text { (induce-type/ min-pos) }\end{array}$ & $\begin{array}{l}\text { Kinase } \\
\text { number }\end{array}$ & $\begin{array}{l}\text { Pfam domain } \\
\text { number }\end{array}$ \\
\hline noise=0 & 10 & 10 \\
\hline induce-cover/9 & 6 & 8 \\
\hline induce-cover/10 & 10 & 9 \\
\hline induce-max /9 & 6 & 10 \\
\hline induce-max/10 & 8 & 8 \\
\hline noise=1 & \multicolumn{2}{|l|}{} \\
\hline induce/8 & 9 & 9 \\
\hline induce/9 & 8 & 8 \\
\hline induce $/ 10$ & 4 & 6 \\
\hline induce $/ 11$ & $\mathbf{1 2}$ & $\mathbf{1 1}$ \\
\hline induce-cover/10 & 7 & 10 \\
\hline induce-cover/11 & 7 & 7 \\
\hline induce-cover/12 & 8 & 4 \\
\hline induce-cover/13 & 8 & 2 \\
\hline induce-cover/14 & $\mathbf{1 2}$ & $\mathbf{1 1}$ \\
\hline induce-max/10 & 11 & 7 \\
\hline induce-max/11 & 7 & 7 \\
\hline induce-max/12 & 8 & 4 \\
\hline induce-max/13 & 10 & 3 \\
\hline induce-max/14 & & \\
\hline
\end{tabular}

\section{DISCUSSION}

ILP has been successfully applied to various areas including bioinformatics (Page and Craven, 2003). Turcotte et al. (2001) used ILP for predicting protein 3D structures. Each protein domain is described by global features (e.g., length, number of helices), adjacency relationships between two consecutive 2D structure elements, and some local properties of the 2D structure elements (e.g., length, presence of some residue). Another ILP application aimed at pharmacophore design for virtual screening purposes (Finn et al., 1998). A pharmacophore is defined as an abstract 3D structure of a molecule that interacts with a protein target. In this case, pairwise distances between atoms or atom groups (e.g., hydrogen donors) of a set of interacting (respectively not interacting) molecules with a specific target are considered for learning. More recently, 3D information on molecules was exploited for searching structurally diverse molecules (drugs) which share a biological activity (Tsunoyama et al., 2008). The considered 3D descriptors are the spatial distances between the atoms composing each active (respectively inactive) molecule of the learning set. Finally, ILP was applied on genomic annotations of proteins coming from public databases (e.g., Pfam, InterPro, PROSITE) in order to predict protein-protein interactions for one specific species (Tran et al., 2005).

To our knowledge, our study is the first which aims to characterize 3D protein-binding sites using ILP. This forms a natural follow-up of previous applications of ILP focusing on the prediction of protein 3D structure, as nowadays protein 3D structures become increasingly available.

As for post-ILP analysis, our approach is innovative and represents a step forward in the interpretation of ILP results in the frame of a knowledge discovery process. Indeed, it offers the expert an effective assistance when exploring the learning results including confrontation with domain knowledge. By facilitating theory interpretation, our approach puts the tricky problem of heuristic parameters selection into perspective. It allows to take benefit from more than one theory. Otherwise, upstream investigation into the effect of numerous parameters on discriminative selection criteria is required as reported in (Turcotte et al., 2001).

We are convinced that our approach can be adapted to other learning problems. Using FCA makes it possible to discover higher-level knowledge units by extracting from the formal concepts first-order logic association rules between the ILP rule bodies (Pasquier et al., 1999). Another perspective of this study concerns the scaling up of ILP programs. Theories can be produced on distinct descriptors subsets corresponding to distinct views on 
the examples. FCA-based joint interpretation of the resulting theories can then enable the discovery of rules involving descriptors from the distinct subsets.

\section{REFERENCES}

Aloy, P., Russell, R., 2003. InterPreTS: Protein Interaction Prediction through Tertiary Structure. Bioinformatics Applications Note, 19 (1): 161-162.

Berman, H.M., Westbrook, J., Feng, Z.,Gilliland, G., Bhat, T.N., Weissig, H., Shindyalov, I.N., Bourne, P.E., 2000. The Protein Data Bank. Nucleic Acids Research, 28: 235-242.

De Raedt L., 2008. Logical and Relational Learning. Springer.

Diella, F., Gould, C.M., Chica, C., Via, A., Gibson, T.J., 2008. Phospho.ELM: a database of phosphorylation sites - update 2008. Nucleic Acids Res., 36 (Database issue): D240-4.

Dubchak, I., Muchnik, I., Mayor, C., Dralyuk, I., Kim, S-H., 1999. Recognition of a protein fold in the context of the SCOP classification. Proteins: Structure, Function, and Genetics, 35(4): 401-407.

Durek, P., Schudoma, C., Weckwerth, W., Selbig, J., Walther, D., 2009. Detection and characterization of 3D-signature phosphorylation site motifs and their contribution towards improved phosphorylation site prediction in proteins. BMC Bioinformatics., 10: 117.

Finn, P., Muggleton, S., Page, D., Srinivasan, A., 1998. Pharmacophore Discovery Using the Inductive Logic Programming System PROGOL. Machine Learning, 30(2-3):241-273.

Ganter, B. and Wille, R., 1999. Formal concept analysis: Mathematical foundations. Springer, Heidelberg, Germany: Springer.

Guharoy, M., Chakrabarti, P., 2005. Conservation and relative importance of residues across protein-protein interfaces. PNAS, 102(43):15447-15452.

Humphrey, W., Dalke, A., Schulten, K., 1996. VMD-Visual Molecular Dynamics. J. Molec. Graphics, 14: 33-38.

Jansen, R., Yu, H., Greenbaum, D., Kluger, Y., Krogan, N.J., Chung, S., Emili, A., Snyder, M., Greenblatt, J.F., Gerstein, M., 2003. A Bayesian networks approach for predicting protein-protein interactions from genomic data. Science, 302(5644): 449-53.

Jones, S., Thornton, J., 1997. Analysis of protein-protein interaction sites using surface patches. J. Mol. Biol., 272: 121-32.

King, R., 2011. Logic, Automation, and the Future of Biology. Proceedings of the Spring School on Modelling Complex Biological Systems, Sophia-Antipolis, France.

Muggleton, S., 1991. Inductive Logic Programming. New Generation Computing, 8(4): 295-318.

Muggleton, S., and De Raedt, L., 1994. Inductive Logic Programming: Theory And Methods. Journal of Logic Programming, 19/20: 629-679.

Obata, T., Yaffe, M.B., Leparc, G. G., Piro, E.T., Maegawa, H., Kashiwagi, A., Kikkawa, R., Cantley L.C., 2000. Peptide and protein library screening defines optimal substrate motifs for AKT/PKB. J. Biol. Chem. 275, 36108-36115.

Page, D., Craven, M., 2003. Biological applications of multi-relational data mining. SIGKDD Explorations, 5(1): 69-79.

Page, D., Srinivasan, A., 2003. ILP: A Short Look Back and a Longer Look Forward. Journal of Machine Learning Research 4: 415-430.

Pasquier, N., Bastide, Y., Taouil, R., Lakhal, L., 1999. Efficient mining of association rules using closed itemset lattices. Journal of Information Systems, 24(1), 25-46.

Punta, M. et al., 2012. The Pfam protein families database. Nucleic Acids Research, 40 (Database Issue): D290-D301.

Smith, G., Sternberg, M., 2002. Prediction of protein-protein interactions by docking methods. Current Opinion in Structural Biology, 12(1):28-35.

Srinivasan, A., 2007. The Aleph Manual. Available at http://www.comlab.ox.ac.uk/oucl/research/areas/machlearn/Aleph/.

Szathmary, L., 2006. Symbolic Data Mining Methods with the Coron Platform. PhD Thesis in Computer Science, Univ. Henri Poincaré - Nancy 1, France.

Tran, T., Satou, K., Ho, T., 2005. Using Inductive Logic Programming for Predicting Protein-Protein Interactions from Multiple Genomic Data. In: Knowledge Discovery in Databases: PKDD 2005; Lecture Notes in Computer Science Volume 3721; Springer Berlin / Heidelberg.

Tsunoyama, K., Ata Amini, A., Sternberg, M., Muggleton, S., 2008. Scaffold Hopping in Drug Discovery Using Inductive Logic Programming. Journal Of Chemical Information And Modeling, 48(5):949-957. 
Turcotte, M., Muggleton, S., Sternberg, M., 2001. Automated discovery of structural signatures of protein fold and function. Journal of Molecular Biology, 306(3):591-605.

Wong, Yh. et al., 2007. Kinasephos 2.0: A Web Server For Identifying Protein Kinase-Specific Phosphorylation Sites Based on Sequences and Coupling Patterns. Nucleic Acids Res., 35 (Web Server issue): W588-W594.

Yu, C.S., Chen, Y.C., Lu, C.H., Hwang, J.K., 2006. Prediction of protein subcellular localization. Proteins, 64: 643-51.

Zhu, H., Domingues, F.S., Sommer, I., Lengauer, T., 2006. NOXclass: prediction of protein-protein interaction types. BMC Bioinformatics, 7: 27.

\section{APPENDIX}

Rule bodies of the theory induce/10/1. Number of covered positive/negative examples are given between square brackets.

p_o(A,29-inf), p_r_distance(A,B,-2,6-8), p_r_distance(A,C,-1,0-6), aliphatic(C). [16,1]

p_r_surface $(A, B, 0), p \_r \_h e l i x(A, C, 21)$, high_polarizability $(C)$. [16,1]

p_r_surface $(A, \operatorname{ser}, 0), p \_r(A, p r o, 1), p \_r \_d i s t a n c e(A, B,-2,0-6), p \_r \_d i s t a n c e(A, C, 2,0-6) .[15,1]$

p_r_helix(A,B,4), p_r_distance $(A, C, 6,8-10), p \_c(A, 105-i n f)$. [15,0]

p_r_helix(A,ser,0), p_r(A,r,-3), p_r_distance $(A, B, 1,0-6)$, medium_polarizability $(B)$. $[15,0]$

p_r(A,pro,1), p_r_distance $(A, B,-3,8-10), p \_r$ distance $(A, C,-1,0-6)$, medium_polarizability $(C)$. $[15,1]$

p_r_helix(A,B,-2), p_r_distance $(A, C, 7,10-11)$, aliphatic(C). [14,1]

p_n $(A, 22-26), p_{-} c(A, 63-75)$. [14,1]

p_r(A,val,-1), p_r_distance $(A, B,-4,0-6), p \_r$ distance $(A, C, 8,0-6) .[14,1]$

p_r_distance $(A, B,-3,10-11), p \_r e s(A, 46-i n f)$. [13,1]

p_r_helix $(A, B, 13)$, small( $B)$, p_r_helix $(A, C, 17)$. $[13,1]$

p_r(A,arg,-2), p_r_distance $(A, B,-1,0-6)$, basic(B). $[13,1]$

p_r_distance $(A, B, 2,6-8), p \_r$ distance $(A, t, 1,0-6)$. $[13,1]$

p_r(A,arg,-3), p_r_distance $(A, B, 3,8-10)$, medium_polarizability(B). [13,1]

p_n(A,26-inf), p_r_sheet(A,B,-4), p_res(A,46-inf). [12,0]

p_r_helix(A,B,27), p_r_distance $(A, C, 9,13-i n f)$. [12,1]

p_r_surface $(A, B, 0), p \_r$ helix $(A$, ser, -6$), p \_r$ distance $(A, C, 2,0-6)$. [12,1]

p_r_distance $(A, B,-10,8-10)$, high_polarizability $(B), p_{\text {D_r_sheet }}(A, B,-10)$. $[11,1]$ 